\title{
An Automatic Wavelet based non-linear Image Enhancement for both Grayscale \& Color Images
}

\author{
Prof. ArjunNichal $^{1}$, Ms.VasudhaPatil ${ }^{2}$, Ms.NehaliPawar ${ }^{3}$, Ms.AshwiniShinde $^{4}$ \\ Assistant Professor, Electronics \& Telecommunication Department, AITRC, Vita, India ${ }^{1}$ \\ Student, Electronics \& Telecommunication Department, AITRC, Vita, India ${ }^{2,3,4}$
}

\begin{abstract}
A proposed wavelet-based dynamic range compression algorithm is used to improve the visual quality of digital images captured in the high dynamic range scenes with non-uniform lighting conditions. The fast image enhancement algorithm which provides dynamic range compression preserving the local contrast and tonal rendition is a very good candidate in aerial imagery applications such as image interpretation for defense and security tasks. This algorithm can further be applied to video streaming for aviation safety. In this paper the latest version of the proposed algorithm which is able to enhance images so that the enhanced images are better than direct human observation, is presented. The results obtained by applying the algorithm to numerous images show strong robustness and high image quality.
\end{abstract}

Keywords: Enhancement, Wavelet based enhancement, Low contrast.

\section{INTRODUCTION}

Images captured from aircrafts, spacecraft's, or satellites usually suffer from lack of clarity, since the atmosphere enclosing Earth has effects upon the images such as turbidity caused by haze, fog, clouds or heavy rain. The visibility of such aerial images may decrease drastically and sometimes the conditions at which the images are taken may only lead to near zero visibility even for the human eyes. Even though human observers may not see much than smoke, there may exist useful information in those images taken under such poor conditions.

Captured images are usually not the same as what we see in a real world scene, and are generally a poor rendition of it. High dynamic range of the real life scenes and the limited dynamic range of imaging devices results in images with locally poor contrast. Human Visual System (HVS) deals with the high dynamic range scenes by Compressing the dynamic range and adapting locally to each part of the scene. There are some exceptions such as turbid (e.g. fog, heavy rain or snow) imaging conditions under which acquired images and the direct observation possess a close parity [1]. The extreme narrow dynamic range of such scenes leads to extreme low contrast in the acquired images.

To deal with the problems caused by the limited dynamic range of the imaging devices, many image processing algorithms have been developed [1]-[4]. These algorithms also provide contrast enhancement to some extent.

Recently we have developed a wavelet-based dynamic range compression (WDRC) algorithm to improve the visual quality of digital images of high dynamic range scenes with non-uniform lighting conditions [5]-[6]. The WDRC algorithm is modified in [7] by introducing an histogram adjustment and non-linear color restoration process so that it provides color constancy and deals with "pathological" scenes having very strong spectral characteristics in a single band. The fast image enhancement algorithm which provides dynamic range compression preserving the local contrast and tonal rendition is a very good candidate in aerial imagery applications such as image interpretation for defence and security tasks. This algorithm can further be applied to video streaming for aviation safety. In this paper application of the WDRC algorithm in aerial imagery is presented. The results obtained from large variety of aerial images show strong robustness and high image quality indicating promise for aerial imagery during poor visibility flight conditions.

\section{METHODOLOGY}

The proposed enhancement algorithm consists of three stages: the first and the third stage are applied in the spatial domain and the second one in the discrete wavelet domain.

\section{A. Histogram Adjustment}

Our motivation in making an histogram adjustment for minimizing the illumination effect is based on some assumptions about image formation and human vision behaviour. The sensor signal $S(x, y)$ incident upon an imaging system can be approximated as the product [2],[7]

$S(x, y)=L(x, y) R(x, y) \ldots \ldots \ldots \ldots \ldots$

Where $R(x, y)$ is the reflectance and $L(x, y)$ is the illuminance at each point $(x, y)$.

In lightness algorithms, assuming that the sensors and filters used in artificial visual systems possess the same nonlinear property as human photoreceptors, i.e., logarithmic responses to physical intensities incident on the their photoreceptors, Equation 1 can be decomposed into a sum of two components by using the transformation $I(x, y)=\log (S(x, y)):$
$I(x, y)=\log (L(x, y))+\log (R(x, y)) \ldots \ldots \ldots .$. 
Where $I(x, y)$ is the intensity of the image at pixel location $(x, y)$

Equation 2 implies that illumination has an effect on the image histogram as a linear shift. This shift, intrinsically, is not same in different spectral bands.

Another assumption of the lightness algorithms is the grayworld assumption stating that the average surface reflectance of each scene in each wavelength band is the same: gray [2]. From an image processing stance, this assumption indicates that images of natural scenes should contain pixels having almost equal average gray levels in each spectral band.

Combining Equation 2 with the gray-world assumption, we perform histogram adjustment as follows:

1) The amount of shift corresponding to illuminance is determined from the beginning of the lower tail of the histogram such that a predefined amount of image pixels is clipped.

2) The shift is subtracted from each pixel value.

3) This process is repeated separately for each color channel.

\section{B. Wavelet Based Dynamic Range Compression and Contrast Enhancement}

\section{Dynamic Range Compression}

Dynamic range compression and the local contrast enhancement in WDRC are performed on the luminance channel. For input color images, the intensity image $I(x, y)$ can be obtained with the following equation:

$I(x, y)=\max [\operatorname{Ii}(x, y)], i \in\{R, G, B\} \ldots \ldots \ldots$

The enhancement algorithm is applied on this intensity image. The luminance values are decomposed using orthonormal wavelet transform as shown in (4)

$$
\begin{gathered}
I(x, y)=\sum_{k, l \in Z} a_{j, k, l} \emptyset_{j, k, l}(x, y)+\sum_{j \geq l} \sum_{k, l \in Z} d_{j, k, l}^{h} \varphi_{j, k, l}^{h}+ \\
\sum_{j \geq l} \sum_{k, l \in Z} d_{j, k, l}^{v} \varphi_{j, k, l}^{v}(x, y)+\sum_{j \geq l} \sum_{k, l \in z} d_{j, k, l}^{v} \varphi_{j, k, l}^{v}
\end{gathered}
$$

Where $a_{j, k, l}$ are the approximation coefficients at scale $J$ with corresponding scaling functions $\emptyset_{j, k, l}(x, y)$ and $d_{j, k, l}$ normalized range $[0,1]$ of $a_{j, k, l}$ to the same range, and is used for compressing the dynamic range represented by the coefficients. The compressed coefficients at level $J$ can be obtained by

$\bar{a}_{j, k, l}=\left[\frac{\sinh \left(4.6248 a_{j, k, l}^{\prime}-2.3124\right)+5}{10}\right]^{r}$

Where $a_{j, k, l}^{\prime}$ are normalized coefficients given by

$a_{j, k, l}^{\prime}=\frac{1}{255} \frac{a_{j, k, l}}{2^{J}} \ldots$

\section{Local Contrast Enhancement}

The local contrast enhancement which employs a center/surround approach is carried out as follows:

The surrounding intensity information related to each coefficient is obtained by filtering the normalized approximation coefficients with a Gaussian kernel
$G(x, y)=K \exp \left(-\frac{x^{2}+y^{2}}{\sigma^{2}}\right) \ldots$

Where $\sigma^{2}$ is surrounds space constant and $\mathrm{K}$ is determined under the constraint that

$\sum_{x} \sum_{y} G(x, y)=1$

Local average image representing the surround is obtained by $2 \mathrm{D}$ convolution of (7) with image $A$ ', the elements of which are the normalized approximation coefficients $a$ ' $J, k, l$ and given by $(6)$ :

$A_{f}(x, y)=A^{\prime}(x, y) * G(x, y)=$

$\sum_{x^{\prime}=0}^{M-1} \sum_{y^{\prime}=0}^{N-1} A^{\prime}\left(x^{\prime}, y^{\prime}\right) G\left(x-x^{\prime}, y-y^{\prime}\right) \ldots \ldots$

The contrast enhanced coefficients matrix Anew which will replace the original approximation coefficients $a J, k, l i s$ given by,

$A_{\text {new }}=\left\{\begin{array}{ll}255 \bar{A}^{R} 2^{J} & \text { for } R \leq 1 \\ 255 \bar{A}^{\frac{1}{R}} & \text { for } R>1\end{array} \ldots \ldots\right.$

where, $R$ is the Centre/surround ratio given by $R=$ $\left(A^{\prime} / A f\right) d, d$ is the enhancement strength constant with a default value of 1 ; is the matrix whose elements are the output of the hyperbolic sine function in (5).

A linear combination of three kernels with three different scales, combined-scale-Gaussian $(G c)$, is used for improved rendition is given by

$$
\begin{gathered}
G_{c}(x, y)=\sum_{k=1}^{3} W_{k} K_{k} \exp \left(-\frac{x^{2}+y^{2}}{\sigma_{k}^{2}}\right), W_{k}=\frac{1}{3} ; k \\
=1 \ldots 3
\end{gathered}
$$

\section{Detail Coefficient Modification}

The detail coefficients are modified using the ratio between the enhanced and original approximation coefficients. This ratio is applied as an adaptive gain mask such as:

$D_{\text {new }}^{h}=\frac{A_{\text {new }}}{A} D^{h} ; D_{\text {new }}^{v}=\frac{A_{\text {new }}}{A} D^{v} ; D_{\text {new }}^{j}=\frac{A_{\text {new }}}{A} D^{j}$

where $A$ and Anew are the original and the enhanced approximation coefficient matrices at level $1 ; D h, D v$, Ddare the detail coefficient matrices for horizontal, vertical and diagonal details at the same level, and Dhnew, $D$ vnew, $D$ dneware the corresponding modified matrices, respectively.

If the wavelet decomposition is carried out for more than one level, this procedure is repeated for each level.

\section{Color Restoration}

A linear color restoration process is used to obtain the final color image in our previous work. For WDRC with color restoration, a non-linear approach is employed. The RGB values of the enhanced color image $I$ enh, $i(x, y)$, along with the $\mathrm{CR}$ factor are given as:

$I_{e n h, i}=\alpha_{i} I_{e n h}, \alpha_{i}=\left(\frac{I_{i}(x, y)}{\max \left(I_{i}(x, y)\right)}\right)^{\beta} \ldots \ldots$ 
Where $I(x, y)$ is the RGB values of the input color image at the corresponding pixel location and $\operatorname{Ienh}(x, y)$ is the resulting enhanced intensity image derived from the inverse wavelet transform of the modified coefficients. Here $\square$ is the non-linear gain factor corresponding. This factor has a canonical value and increases the color saturation resulting in more appealing color rendition.

Since the coefficients are normalized during the enhancement process, the enhanced intensity image obtained by (15) the inverse transform of enhanced coefficients, along with the enhanced color image given by span almost only the lower half of the full range of the histogram. For the final display domain output Ienh, $i$ 's in (15) are stretched to represent the full dynamic range. Histogram clipping from the upper tail of histograms in each channel give the best results in converting the output to display domain.

\section{RESULT ANALYSIS AND DISCUSSION}

\section{A. Quality Parameter:}

\section{Peak Signal to noise ratio:}

PSNR term is mainly used to measure the quality of reconstruction of lossy compression. It is mostly defined through MSE(mean square error).PSNR is basically expressed in the logarithmic decibel scale.The PSNR (in $\mathrm{dB}$ ) is defined as:

Where

$$
P S N R=10 . \log _{10}\left(\frac{M A X_{I}^{2}}{M S E}\right)
$$

$$
M S E=\frac{1}{m n} \sum_{i=0}^{m-1} \sum_{j=0}^{n-1}[I(i, j)-K(i, j)]^{2}
$$

\section{Mean Square Error:}

The MSE is the cumulative squared error between the compressed and the original image, whereas PSNR is a measure of the peak error.

$$
M S E=\frac{1}{m n} \sum_{i=0}^{m-1} \sum_{j=0}^{n-1}[I(i, j)-K(i, j)]^{2}
$$

\section{Absolute Mean brightness Error:}

The proposed method is trying to preserve brightness mean more and more possible by considering value ofabsolute mean brightness error (AMBE). AMBE is calculated from equation below.

$$
A M B E=|E[Y]-E[X]|
$$

Where $E[Y]$ and $E[X]$ are mean of new and original gray level of image, respectively.

\section{B. Results}

The proposed algorithm has been applied to numerous images with different degree of turbidity. The resultsshowimproved clarity i.e. the increased visibility distance forhaze, fog, clouds and heavy rain. The algorithm works well forimages captured in diverse flight conditions.

\section{(A) For grayscale image:}

Fig 1.(a) shows low contrast image, after applying WDRC algorithm we get enhanced image as shown in fig 1(b).

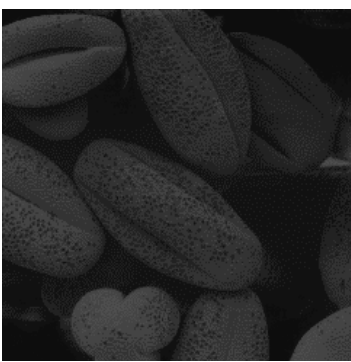

(a)

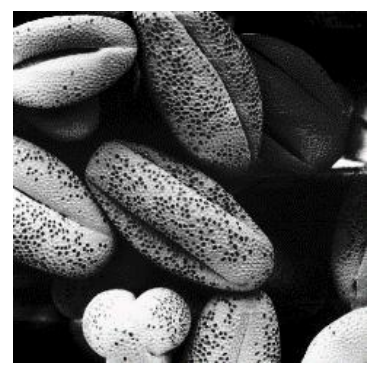

(b)
Figure 1. (a) Original low contrast bean image, (b) Enhanced Bean Image

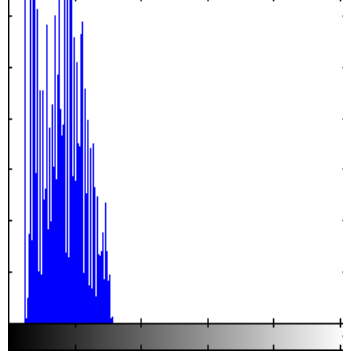

(c)

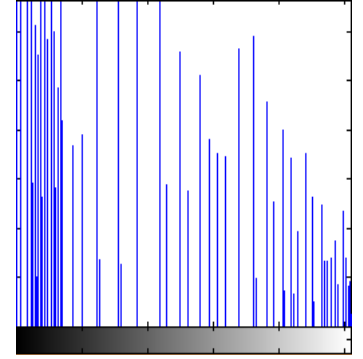

(d)
Graph 1.(c)Histogram for Original low contrast bean image,(d)Histogram for Enhanced Bean Image

\begin{tabular}{|l|l|l|}
\hline \multicolumn{3}{|l|}{ Parameter } \\
\hline PSNR & MSE & AMBE \\
\hline 58.6385 & 0.0889674 & 41.7002 \\
\hline
\end{tabular}

\section{(B) For Color image}

Fig 2.(a) shows low contrast image, after applying WDRC algorithm we get enhanced image as shown in fig 1(b).

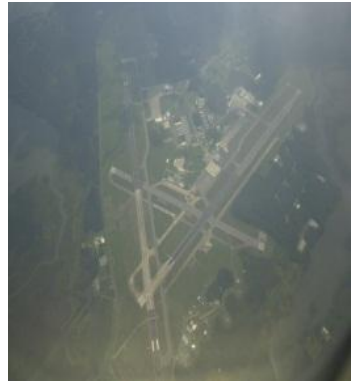

(a)

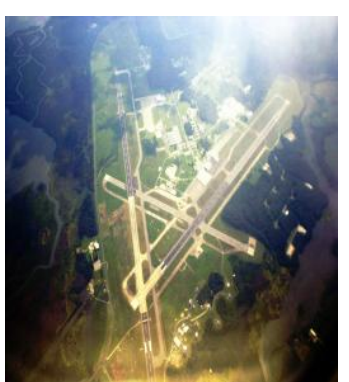

(b)
Figure 2. (a) Original low contrast airport image, (b) Enhanced airport Image

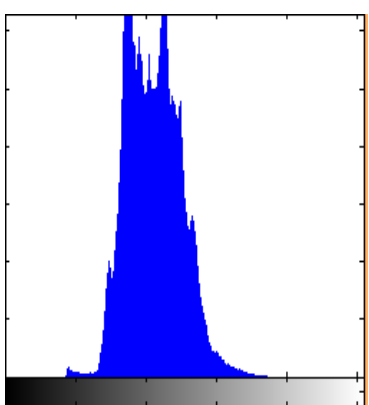

(c)

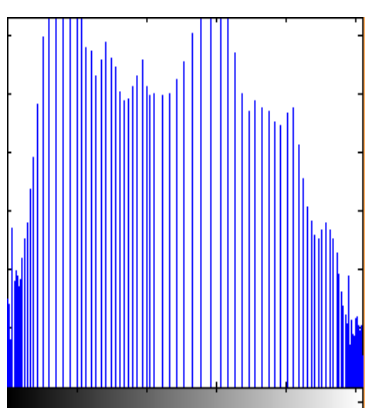

(d)
Graph 2. (c)Histogram for Original low contrast airport image, (d)Histogram for Enhanced airport Image 
INTERNATIONAL JOURNAL OF INNOVATIVE RESEARCH IN ELECTRICAL, ELECTRONICS, INSTRUMENTATION AND CONTROL ENGINEERING Vol. 3, Issue 4, April 2015

\begin{tabular}{|l|l|l|}
\hline \multicolumn{3}{|l|}{ Parameter } \\
\hline PSNR & MSE & AMBE \\
\hline 61.6757 & 0.0443219 & 5.8005 \\
\hline
\end{tabular}

Fig 2.(a) shows low contrast image, after applying WDRC algorithm we get enhanced image as shown in fig 1(b).

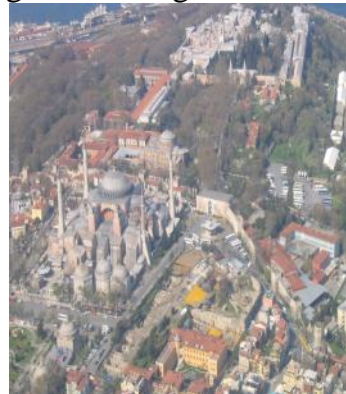

(a)

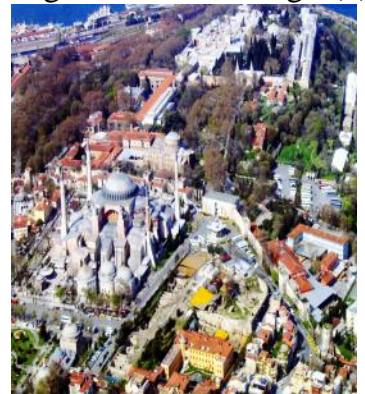

(b)
Figure 3. (a) Original low contrast town image, (b) Enhanced town Image

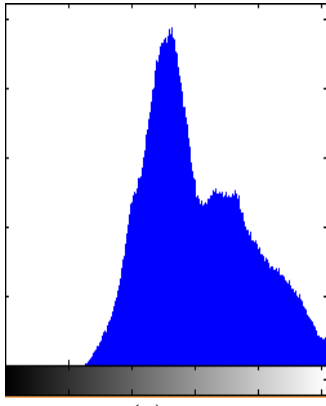

(c)

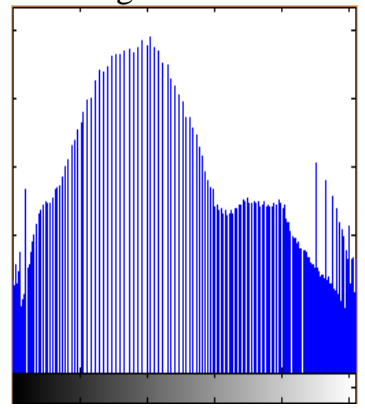

(d)
Graph 3. (c)Histogram for Original low contrast town image, (d)Histogram for Enhanced town Image

\begin{tabular}{|l|l|l|}
\hline \multicolumn{3}{|l|}{ Parameter } \\
\hline PSNR & MSE & AMBE \\
\hline 63.0533 & 0.0327088 & 21.1577 \\
\hline
\end{tabular}

\section{MATLAB Implementation:}

Following Graphical user Interfaces shows the MATLAB implementation of proposed scheme.

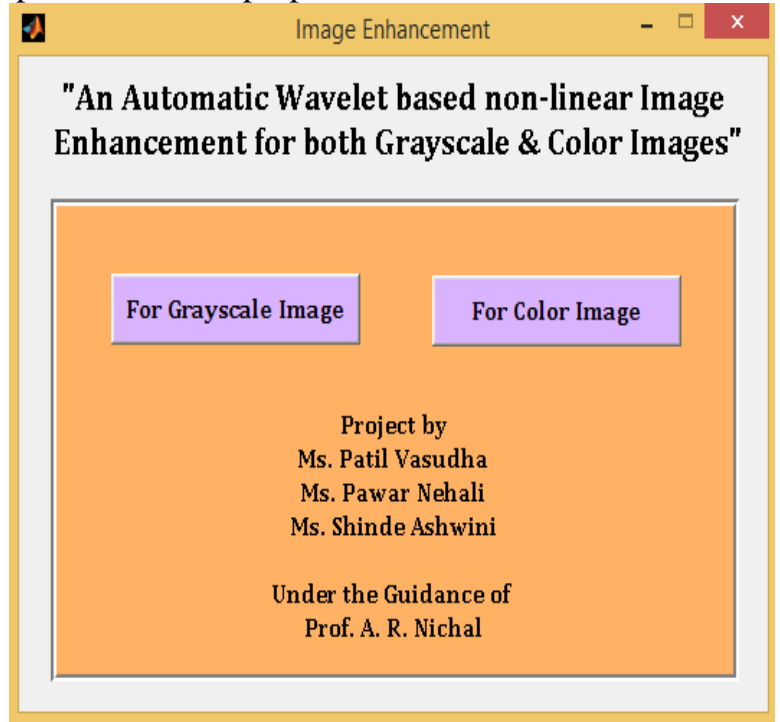

Figure 4. Main GUI window

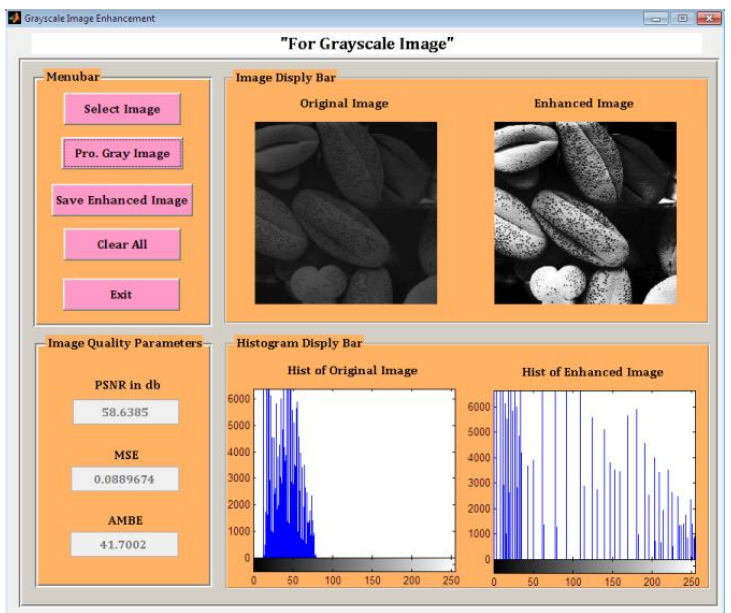

Figure 5. Grayscale Image Enhancement

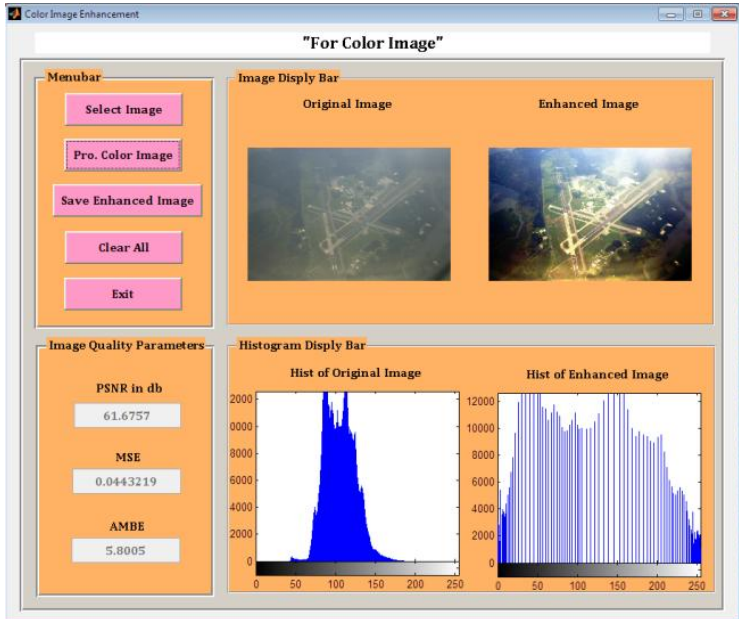

Figure 6. Color Image Enhancement

\section{CONCLUSION}

In this paper application of the WDRC algorithm in aerial imagery is presented. The results obtained from large variety of images show strong robustness, high image quality, and improved visibility indicating promise for images during poor visibility flight conditions. This algorithm can further be applied to real time video streaming and the enhanced video can be projected to the pilot's heads-up display for aviation safety.

\section{REFERENCES}

[1] S. M. Pizer, J. B. Zimmerman, and E. Staab, "Adaptive grey level assignment in CT scan display," Journal of Computer Assistant Tomography, vol. 8, pp. 300-305,(1984).

[2] A. Hurlbert, "Formal Connections Between Lightness Algorithms", Journal of the Optical Society of America, vol. 3, No 10 pp. 16841693, (1986).

[3] D. J. Jobson, Z. Rahman, and G. A. Woodell, “A multi-scale retinex for bridging the gap between colorimages and the human observation of scenes," IEEE Transactions on Image Processing, Vol. 6, pp. 965-976, (1997).

[4] K. Barnard and B. Funt, "Analysis and improvement of multi-scale retinex," Proc. IS\&T/SID Color Imaging Conference: Color Science, Systems and Applications, pp. 221-226, (1997).

[5] H. Ngo, L. Tao and K. V. Asari, "Design of an efficient architecture for real-time image enhancement based on a luma-dependent nonlinear approach," Proc. IEEE International Conference on Information TechnologyTechnology: Coding and Computing ITCC'04, Vol. 1, pp. 656-660,( 2004) 
[6] L. Tao and K. V. Asari, "An adaptive and integrated neighborhood dependent approach for nonlinear enhancement of color images," SPIE Journal of Electronic Imaging, Vol. 14, No. 4, ,pp. 1.1-1.14. (2005)

[7] L. Tao, R. C. Tompkins, and K. V. Asari, "An illuminancereflectance model for nonlinear enhancement of video stream for homeland security applications," IEEE International Workshop on Applied Imagery and Pattern Recognition, AIPR - 2005, Washington DC, October 19 - 21, (2005)

[8] N. Unaldi, K. V. Asari, Z. Rahman, "Fast and robust wavelet-based dynamic range compression with local contrast enhancement", SPIE proc., Vol. 6978, (2008)

[9] N. Unaldi, P. Sankaran, K. V. Asari, Z. Rahman, "Image enhancement for improving face detection under non-uniform lighting conditions", ICIP 2008. 15th IEEE International Conference on Image Processing, (2008)

[10] N. Unaldi, K. V. Asari, Z. Rahman, "Fast and robust wavelet-based dynamic range compression and contrast enhancement model with color restoration", SPIE proc.,(2009) in press.

[11] B. K. P. Horn, "Determining Lightness from an image", Computer Graphics and Image Processing, vol. 3, no. 1, pp.277-299,(1974)

[12] D. J. Jobson, Z. Rahman, G.A. Woodell, "Statistics of visual representation," SPIE Proc., Vol. 4736, pp. 25-35. (2002)

[13] R. C. Gonzalez, R. E. Woods, Digital Image Processing Using MATLAB, 2nd Ed. Prentice Hall, pp. 380-400 (2002)

[14] D. J. Jobson, Z. Rahman, G. A. Woodell, G.D.Hines, "A Comparison of Visual Statistics for the Image Enhancement of FORESITE Aerial Images with Those of Major Image Classes," Visual Information Processing XV, Proc. SPIE 6246, (2006)

[15] J. B. Zimmerman, S. B. Cousins, K. M. Hartzell, M. E. Frisse, and M. G. Kahn, "A psychophysical comparison of two methods for adaptive histogram equalization," Journal of Digital Imaging, vol. 2, pp. 82-91 (1989)

[16] Z. Rahman, D. J. Jobson, and G. A. Woodell, "Retinex Processing for Automatic Image Enhancement", Journal of Electronic Imaging, January (2004)

\section{BIOGRAPHIES}

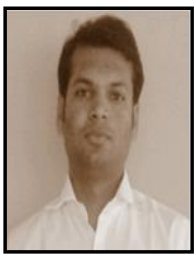

Prof.Arjun Nichal Received his M.tech degree from walchand college of Engg sangli in 2012. Pursuing PHD from Shivaji University Kolhapur. Working as a assistant professor in AITRC vita. His area of interest is image processing, embedded system. Published one E-book and 11 international journal papers.

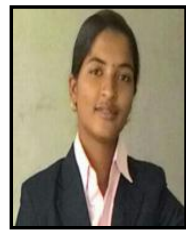

Ms.Ashwini Shinde Pursuing her BE in Electronics \& Telecommunication from AITRC vita. Her area of interest is Image Processing and Embedded system.

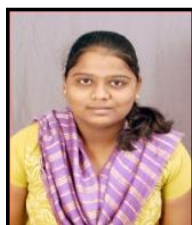

Ms.Vasudha Patil Pursuing her BE in Electronics \& Telecommunication from AITRC vita. Her area of interest is Image Processing and Embedded system.

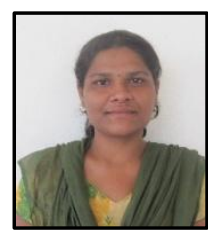

Ms.Nehali Pawar Pursuing her BE in Electronics \& Telecommunication from AITRC vita. Her area of interest is Image Processing and Embedded system. 